\title{
Co-segmentation of Functional and Anatomical Images
}

\author{
Ulas Bagci ${ }^{1}$, Jayaram K. Udupa ${ }^{2}$, Jianhua $\mathrm{Yao}^{1}$, and Daniel J. Mollura ${ }^{1}$ \\ 1 Center for Infectious Diseases Imaging, Department of Radiology and Imaging \\ Sciences, National Institutes of Health (NIH), Bethesda, USA \\ 2 Department of Radiology, University of Pennsylvania, Philadelphia, PA, USA
}

\begin{abstract}
This paper presents a novel method for segmenting functional and anatomical structures simultaneously. The proposed method unifies domains of anatomical and functional images (PET-CT), represents them in a product lattice, and performs simultaneous delineation of regions based on a random walk image segmentation. In addition, we propose a simple yet efficient object/background seed localization method, where background and foreground object cues are automatically obtained from PET images and propagated onto the corresponding anatomical images (CT). In our experiments, abnormal anatomies on PET-CT images from human subjects are segmented synergistically by the proposed fully automatic co-segmentation method with high precision (mean DSC of $91.44 \%$ ) in seconds (avg. 40 seconds).
\end{abstract}

Keywords: Joint Segmentation, PET-CT, Random Walk, Object Detection.

\section{Introduction}

Hybrid imaging modalities such as PET-CT and MRI-PET are in vogue since they can achieve higher sensitivity and specificity than the component modalities alone [1. As a functional measurement, standardized uptake value (SUV) is often used in Positron Emission Tomography (PET) imaging (See Figs. 17-e). However, SUVs alone are not enough in a PET-CT acquisition to diagnose, characterize, and stage the disease, since anatomic boundaries of the corresponding structure on Computed Tomography (CT) are also needed for this calculation. As a result, diagnostic sensitivity and specificity achieved in hybrid imaging modalities (i.e., PET-CT) are higher than either modality alone. All these processes require precise segmentations of both PET and CT images. There are several reasons why region delineations need to be performed meticulously and accurately in both CT and PET images. (1) errors in segmentation can distort the SUV calculations by altering the region's margins, (2) manual or semi-automated mechanisms of segmentation can be inefficient and suffer from unacceptable inter-observer variance, and (3) using CT for segmenting lesions that are quantitatively measured on PET can overlook and exclude other important quantitative data such as texture features in PET images. Therefore, we

N. Ayache et al. (Eds.): MICCAI 2012, Part III, LNCS 7512, pp. 459-467, 2012.

(C) Springer-Verlag Berlin Heidelberg 2012 
aim to produce an automated, efficient, and reproducible segmentation method that simultaneously unifies anatomic and functional information. The proposed method can be especially useful in quantifying lesions characterized by fuzzy boundaries and low contrast from surrounding normal structures.

Related Works: Except for a few studies [2, 3], radiotracer uptake regions are usually delineated manually in clinical routines. Fixed and adaptive thresholding and region based segmentation methods (i.e., fuzzy c-means, region growing, and watershed) are also used to determine boundaries [4. However, segmentations of PET images in these studies are formulated without incorporating corresponding anatomical information (i.e., CT), and the accuracy, robustness, and reproducibility of these methods are suspect in more difficult cases.

A joint PET-CT image segmentation method was proposed recently in [2], where a Markov Random Field (MRF) algorithm was formulated on a graph. The method requires user interaction, and it was used only in images from headneck with large tumors. Its performance in small uptake regions was not assessed. Another problem was due to the potentially unrealistic assumption that there is a one-to-one correspondence between PET and CT delineations. For example, lesions may have smaller uptake regions (on PET images) compared to outlines of lesions in CT images because of functional or metabolic characteristics of the tumor. In this study, we consider these issues and propose a co-segmentation method which is driven by the uptake regions from PET in finding the correct anatomical boundaries in the corresponding CT images. Our algorithm also uses a novel automatic background/foreground seed localization technique to make the whole system fully automatic.

\section{Methods}

Graph theoretic segmentation methods represent space elements (spels for short) of an image as a graph with spels as its nodes and edges defined by spel adjacency with cost values assigned to edges. These methods partition the nodes into two disjoint subsets representing the object and background. This process can be accomplished by finding the minimum cost/energy among all possible cut scenarios in the graph (as in graph-cut algorithms) or optimizing some sort of discrete energies combining boundary regularization with regularization of regional properties of segments [2]. However, a common problem with these is the "small cut" behavior. As a possible solution to this behavior, the random walk algorithm is more efficient than the conventional graph-cut algorithms in terms of handling ambiguities among object boundaries (i.e., weak edges among objects) and more accurate segmentations in noisy and low contrast images [5]. Since PET images are low resolution, and weak boundaries often exist in CT images, random walk segmentation is a natural choice for the simultaneous segmentation of PET and CT images. We reformulate random walk as a co-segmentation algorithm for delineating PET and CT images simultaneously and providing globally optimum segmentation results. To create a fully automated framework, we propose an automatic seed localization system by identifying interesting uptake regions (IUR) 
from PET images, using these regions to identify foreground and background seeds, and propagating the detected background and foreground seeds to the corresponding CT images.

\subsection{Automated Random Walk Co-segmentation}

Let a connected and undirected graph $G$ be represented as a pair $G=(V, E)$ with vertices/nodes $v \in V$ and edges $e \in E \subseteq V \times V$. Conventionally, a node $v_{i}$ is said to be a neighbor of another node $v_{j}$ if they are connected by an edge $e_{i j}$, which is weighted by $w_{i j}$. Since the graph is assumed to be connected and undirected: $w_{i j}=w_{j i}$. By following the recommendation in [5], we construct the weighting functions for PET and CT image modalities separately as

$$
\begin{aligned}
w_{i j}^{P E T} & =\exp \left(-\beta^{P E T}\left(I_{i}^{P E T}-I_{j}^{P E T}\right)^{2}\right), \\
w_{i j}^{C T} & =\exp \left(-\beta^{C T}\left(I_{i}^{C T}-I_{j}^{C T}\right)^{2}\right) .
\end{aligned}
$$

where $I_{i}$ indicates the intensity at spel $i$, and $\beta$ represents a weighting factor. Note that PET and CT images are obtained from the same scanner in the same scanning session; therefore they are registered so that there is a one-to-one spel correspondence between them. Conventionally, the desired random walker probabilities have the same solution as the combinatorial Drichlet problem [6]: $D[x]=\frac{1}{2} x^{T} L x$, where $x$ denotes the probability (potential) assumed at each node [5], and $L$ represents combinatorial Laplacian matrix. For each of the PET and CT modalities, this matrix can be formulated as:

$$
L_{i j}^{\mathcal{X}}= \begin{cases}d_{i}^{\mathcal{X}} & \text { if } i=j \\ -w_{i j}^{\mathcal{X}} & \text { if } v_{i}^{\mathcal{X}} \text { and } v_{j}^{\mathcal{X}} \text { are adjacent nodes } \\ 0 & \text { otherwise. }\end{cases}
$$

where $\mathcal{X}$ is either PET or CT, and $d_{i}$ is the degree of a vertex for all edges $e_{i j}$ incident on $v_{i}$ and is defined as: $d_{i}=\sum_{e_{i j} \in E} w\left(e_{i j}\right)$. Moreover, $v^{P E T}$ and $v^{C T}$ are the nodes pertaining to the graph constructed on PET and CT images, respectively.

Simultaneous segmentation of PET and CT images on the graph requires a special representation of both data without losing information. From graph theory, it has been well known that given two graphs and their product as an outcome graph, an edge exists in the product graph if and only if an edge exists in both graphs [6]. Defining a special graph combining these two graphs, or a hypergraph, is a natural choice to satisfy this property. Given two graphs $G^{C T}=\left(V^{C T}, E^{C T}\right)$ and $G^{P E T}=\left(V^{P E T}, E^{P E T}\right)$, without loss of generality, we define our special product graph as $G^{f u s e}=\left(V^{f u s e}, E^{f u s e}\right)$. Note that $V^{C T}$ and $V^{P E T}$ have the same number of spels due to one-to-one spell correspondence. $G^{\text {fuse }}$ has an important property that an edge exists in $E^{\text {fuse }}$ if and only if the corresponding nodes are adjacent in both $G^{C T}$ and $G^{P E T}$. Thus, 


$$
\begin{array}{r}
V^{\text {fuse }}=\left\{\left(v_{i}^{C T}, v_{i}^{P E T}\right): v_{i}^{C T} \in V^{C T} \wedge v_{i}^{P E T} \in V^{P E T}\right\}, \\
E^{f u s e}=\left\{\left(\left(v_{i}^{C T}, v_{i}^{P E T}\right),\left(v_{j}^{C T}, v_{j}^{P E T}\right)\right):\right. \\
\left.\left(v_{i}^{C T}, v_{j}^{C T}\right) \in E^{C T} \wedge\left(v_{i}^{P E T}, v_{j}^{P E T}\right) \in E^{P E T}\right\} .
\end{array}
$$

We use the product graph $G^{\text {fuse }}$ to segment objects simultaneously instead of using separate implementation of $G^{C T}$ and $G^{P E T}$. Since the critical points of $D[x]$ are assumed to be minima, finding these minima points yields the solution for the random walk probabilities. This requires an updated definition of the combinatorial Laplacian matrix on the product graph. Namely, the combinatorial Laplacian matrix $\left(L^{\text {fuse }}\right)$ of the product graph $G^{\text {fuse }}$ is defined as $L^{\text {fuse }}=$ $\left(L^{C T}\right)^{\alpha} \otimes\left(L^{P E T}\right)^{\theta}$, for some constants $0 \leq \alpha, \theta \leq 1$. Meanwhile, the initial probability distribution $x^{f u s e}$ of the product graph is $x^{f u s e}=\left(x^{C T}\right)^{\zeta} \otimes\left(x^{P E T}\right)^{\eta}$, where $\zeta$ and $\eta$ are used to optimize the initial probability distributions subject to the constraint $0 \leq \zeta, \eta \leq 1$, and $x^{C T}$ and $x^{P E T}$ denote initial probability distributions (i.e., priors) over nodes of $G^{C T}$ and $G^{P E T}$. Performing a random walk on the product graph $G^{f u s e}$ is equivalent to performing a simultaneous random walk on the graphs $G^{C T}$ and $G^{P E T}$ [6]. Therefore, the combinatorial formulation of Drichlet integral can be re-written as

$$
D\left[x^{\text {fuse }}\right]=\frac{1}{2}\left(x^{\text {fuse }}\right)^{T} L^{\text {fuse }} x^{\text {fuse }}=\frac{1}{2} \sum_{e_{i j} \in E^{f u s e}} w_{i j}^{\text {fuse }}\left(x_{i}^{f u s e}-x_{j}^{\text {fuse }}\right)^{2},
$$

where a combinatorial harmonic function of $x^{\text {fuse }}$, satisfying the Laplace equation $\nabla^{2} x^{\text {fuse }}=0$, minimizes Eqn. 5. We can decompose Eqn. 5 ] by considering prior probabilities and Laplacian matrices of labeled and unlabeled nodes separately as

$$
D\left[x_{u}^{f u s e}\right]=\frac{1}{2}\left[\left(x^{f u s e}\right)_{l}^{T}\left(x^{f u s e}\right)_{u}^{T}\right]\left[\begin{array}{cc}
L_{l}^{f u s e} & B \\
B^{T} & L_{u}^{f u s e}
\end{array}\right]\left[\begin{array}{l}
x_{l}^{f u s e} \\
x_{u}^{f u s e}
\end{array}\right]
$$

where $B$ corresponds to the sub-matrix in the matrix decomposition of $L^{\text {fuse }}$. Given the fact that the combinatorial Laplacian matrix $L^{\text {fuse }}$ is positive semidefinite, critical points of $D\left[x^{\text {fuse }}\right]$ are only the minima, hence, differentiating $D\left[x^{\text {fuse }}\right]$ with respect to prior probability distributions of unlabeled nodes $x_{u}^{\text {fuse }}$ and finding the minima yields $L_{u}^{\text {fuse }} x_{u}^{\text {fuse }}=-B^{T} x_{l}^{\text {fuse }}$, where $L_{u}^{\text {fuse }}$ and $B$ are known, and $x_{l}^{\text {fuse }}$ is the prior for labeled node. Solving this equation for every $x_{u}^{f u s e}$ completes the binary labeling problem of co-segmentation on the graph $G^{\text {fuse }}$.

\subsection{Automated Seed Localization}

The goal in seed localization is to define the foreground and background regions in both PET and CT images. An overview of the proposed automated background and foreground seed localization is sketched in Fig. 1(f-i). Briefly, we 

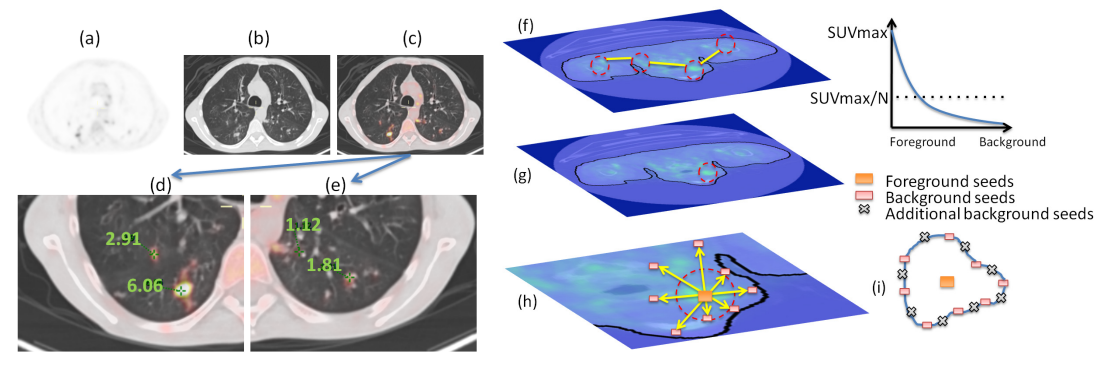

Fig. 1. Even though intensity profiles in CT images (b) show similar characteristics, SUV from PET images (a) might further characterize the nodules. Radioactivity uptake regions are shown in small nodules in (a and c)(and details of fused image (c) are shown in $\mathrm{d}$ and e). The concepts of interesting uptake region (IUR) detection and background/foreground seed localization are sketched in (f-i).

partition the image into a set of regions such that some of those regions (i.e., IURs) are more similar to each other than to those of other regions. We accomplish this selection procedure by defining an encoder function $c($.$) , which is$ nothing but a threshold interval for PET images:

$$
c(\lambda)=\left\{\begin{array}{lr}
1, \lambda \in\left[S U V_{\max }^{\text {global }} / N, S U V_{\text {max }}^{\text {global }}\right], \\
0, & \text { otherwise }
\end{array}\right.
$$

where $(N>1) \in \mathcal{R}$ is free parameter, and each region identified by the encoding function is considered as IUR. Once IURs are identified, we set the number of disconnected IURs as a hard constraint (i.e., number of objects to be segmented) on seed localizations. The seed localization procedure is as follows: (1) Both CT and PET images are median filtered to smooth the images. (2) We find the skin boundary from CT scan by using simple mathematical morphology (i.e., a few times opening followed by closing) and incorporate this information into the corresponding PET image (black outlines in Fig. 1f). (3) We find the IURs inside the body region and pertaining to the interval of $\left[S U V_{\max }^{\text {global }} / N, S U V_{\text {max }}^{\text {global }}\right]$ by using Eqn. 7 (Fig. 1f). (4) For each IUR, the spels with the maximum SUVs $\left(S U V_{\text {max }}^{\text {local }}\right)$ of that particular IUR are marked as foreground seeds (Fig. 18). Note that maximum SUV of one particular IUR (i.e., $S U V_{\text {max }}^{\text {local }}$ ) does not necessarily equal $S U V_{\max }^{\text {global }}$. (5) At each spel, marked as a foreground seed, we explore its neighborhood through an 8-connectivity graph labeling algorithm [6]. For all 8-directions starting from each foreground seed, we find locations of the very first spels with values less than or equal to the $S U V_{\text {max }}^{\text {global }} / N$. Those spels are marked as background seeds. Fig. 1h and i illustrate this procedure for a particular foreground seed. (6) We add additional background seeds into the spels lying in the spline connecting background seeds determined in the previous step (Fig. 11). Fig. 2 shows automatically located seeds with (b) and without (a) additional background seeds. We find step 6 necessary to avoid any leakage in delineation of the abnormal anatomy in CT images. Fig. 2r shows an example of 


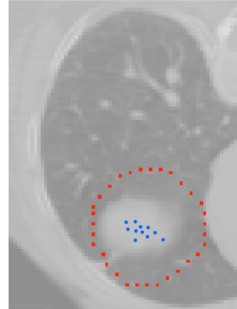

(a)

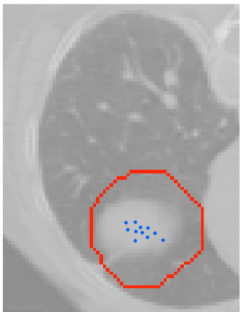

(b)

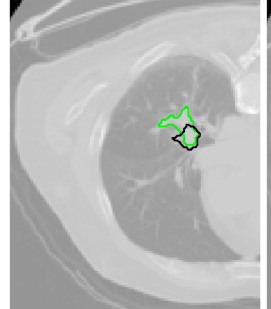

(c)

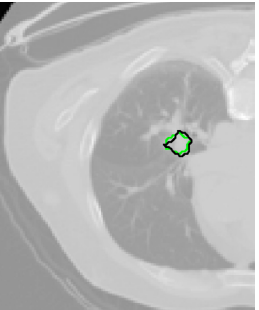

(d)

Fig. 2. (a) Automatically located background (red) and foreground (blue) seeds. (b) Additional background seeds are obtained by connecting initial background seeds using b-splines. (c) Ground truth (black) and random walk segmentations using only a limited number of background seeds. (d) The effect of the proposed seed localization method (with additional background seeds) in avoiding possible leakages.

the leakage occurring due to the close proximity of normal and abnormal tissues with similar intensity profiles while the effect of having additional background seeds on segmentation is shown in Fig. $2 \mathrm{~d}$.

\section{Results}

Data and Evaluation Metrics: A retrospective study involving 15 patients with PET-CT scans was performed. The resolution of PET scans is limited to spels of size $4 \mathrm{~mm}$ x $4 \mathrm{~mm}$ x $4 \mathrm{~mm}$. The patients considered have infectious lung disease abnormality patterns including ground glass opacities, consolidations, nodules, tree-in-bud, lung tumors, and non-specific lung lesions. While PET scans consist of more than 300 slices per patient, CT scans have the same number of slices but different in-plane resolution with $0.98 \mathrm{~mm}$ x $0.98 \mathrm{~mm} \times 1.5 \mathrm{~mm}$ spel size. Dice similarity coefficients (DSC) and Hausdorff distance (HD) are used to evaluate the accuracy of segmentations. High DSC and low HD values indicate goodness of the image segmentation method. The ground truth segmentations were obtained from manual delineations of two expert interpreters on PET-CT data, and average DSC values were reported.

Qualitative and Quantitative Evaluation: Fig. 3] shows segmentation examples. Co-segmentation of PET-CT images (blue), segmentation of CT images (green), and PET images (yellow) are shown in first, second, and third columns, respectively. The fourth column reveals all segmentations overlaid together for comparison. In all images, ground truth segmentations are shown in black. It can be seen that the co-segmentation results are superior to delineation using only CT and only PET images, and agree well with the ground truth delineations. Fig. 4 reports the average DSCs and HDs of delineations over 15 subjects and comparison to the method presented in [2]. Note that co-segmentation on PETCT images is superior in accuracy of segmentations to other methods. 


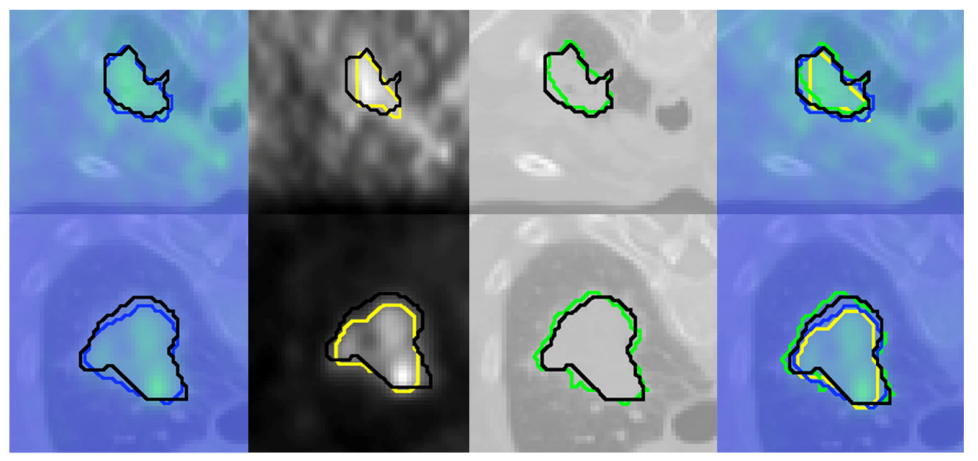

Fig. 3. Two different segmentation examples of uptake regions are shown in each column. First column: co-segmentation (blue) and ground truth (black) are overlaid. Second column: ground truth (black) and segmentation from PET only (yellow). Third column: ground truth (black) and segmentation from CT only (green). Fourth column: all segmentations and ground truth are overlaid together.
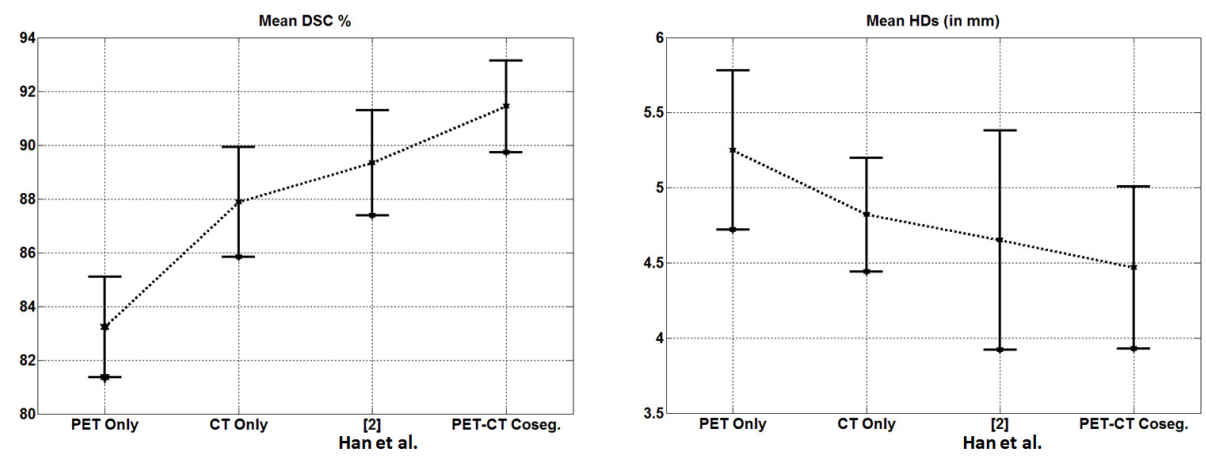

Fig. 4. Mean DSCs and HDs are enlisted. DSC ratios: PET Only: $83.23 \mp 1.87, \mathrm{CT}$ Only: $87.88 \mp 2.04$, Han et al.: $89.34 \mp 1.95$, PET-CT cosegm.: $91.44 \mp 1.71$. HDs ratios: PET Only: $5.25 \mp 0.53$, CT Only: $4.82 \mp 0.38$, Han et al: $4.65 \mp 0.73$, PET-CT cosegm.: $4.47 \mp 0.54$.

Training, Parameter Selection, and Computational Issues: First, we upsampled the PET images so that the PET and CT images have the same size, and each spel in PET has its correspondence in CT in the same spatial coordinate. We used a training set consisting of PET-CT scans of 5 patients (different from the test data set) to train parameters of the segmentation process explained below. In defining the product graph, we weight the corresponding combinatorial Laplacian matrices $\left(L^{C T}\right.$ and $L^{P E T}$ ) with $\alpha$ and $\theta$ and the initial probability distributions $x^{C T}$ and $x^{P E T}$ with $\zeta$ and $\eta$, respectively. We noted that multiplication and summation of Laplacian matrices do not have significant effects on the segmentation results if $\zeta$ and $\eta$ are set to 1 . The best possible 
combination of $(\alpha, \theta)$ is found to be $(0.2,0.8)$ when $\zeta=\eta=1$. Other parameters were set to: $\sigma^{P E T}=\sigma^{C T}=1, \beta^{P E T}=0.3$, and $\beta^{C T}=0.7$. Lastly, based on our experimental results and empirical observations, we set a slightly tighter bound on $S U V_{\max } / N$ by setting $N=2$ compared to the conventional clinical usage where $N$ is usually 2.5. The combined running time for detecting all seeds and delineating the corresponding binary labeling problem per slice does not exceed 2 seconds at maximum, and averages 0.8 seconds. The maximum number of slices showing high tissue activity does not exceed 50, therefore all detection and segmentation procedures end within an average of 40 seconds (using an Intel (R) workstation with Core(TM) i7 CPU 930 running at $2.80 \mathrm{GHz}$ with $12 \mathrm{~GB}$ RAM). The proposed algorithm can be run in either pseudo-3D or 3D directly.

\section{Discussion and Conclusion}

We proposed a joint-segmentation framework for anatomical and functional images. Our approach differs from the approach reported in [2] in the following manners: (1) We propose a completely automated method of segmentation, therefore user-interaction is not required. As a result, the proposed method is faster and reproducible. (2) We do not have constraints about one-to-one correspondence of abnormal anatomy in PET and CT images, as it is possible that a tumor can reveal increased radioactivity uptake only in some areas inside the tumor region, or it may be possible for an uptake region to enclose both normal and abnormal anatomy in CT scans. Instead, it is semantically more meaningful and reliable to drive the segmentation of both images based on the guidance of PET images. (3) Although MRF based segmentation of images on graphs is shown to be useful in many applications, incorporating an additional energy term, similar to the one proposed in 2, may not be the best solution for avoiding the leakage in CT and PET delineations. In contrast, the proposed method is more powerful in terms of handling image noise and low contrast and therefore more suited to PETCT segmentations. Another advantage of our method is the special graph model constructed by a product graph via taking into account the anatomical and functional image features to derive algorithms for accurately finding the most likely IUR boundaries. Initial results on 15 clinical PET-CT images (i.e., thousands slices) show high accuracy in delineations by the proposed method. Furthermore, the proposed method considerably reduces the time for manual segmentations. As an extension of this work, we aim to generalize the proposed co-segmentation method for MRI-PET and multi-fusion MRI-CT-PET segmentations.

\section{References}

[1] Judenhofer, M.S., et al.: Simultaneous PET-MRI: a new approach for functional and morphological imaging. Nature Medicine 14, 459-465 (2008)

[2] Han, D., Bayouth, J., Song, Q., Taurani, A., Sonka, M., Buatti, J., Wu, X.: Globally Optimal Tumor Segmentation in PET-CT Images: A Graph-Based Cosegmentation Method. In: Székely, G., Hahn, H.K. (eds.) IPMI 2011. LNCS, vol. 6801, pp. 245-256. Springer, Heidelberg (2011) 
[3] Montgomery, D.W., et al.: Fully automated segmentation of oncological PET volumes using a combined multiscale and statistical model. Medical Physics 34(2), 722-736 (2007)

[4] Jentzen, W., et al.: Segmentation of PET Volumes by Iterative Image Thresholding. J. Nucl. Med. 48, 108-114 (2007)

[5] Grady, L.: Random Walks for Image Segmentation. IEEE Transactions on Pattern Analysis and Machine Intelligence 28(11), 1768-1783 (2006)

[6] Harary, F.: Graph Theory. ABP Publishing (1994) 